\title{
COMPUTER SIMULATION OF THE BEAM-BEAM INTERACTION AT A CROSSING ANGLE
}

\author{
A. Piwinski* \\ LEP Division, CERN, \\ Geneva, Switzerland
}

\section{Abstract}

The simulation is done for protons in HERA which was first designed with a crossing angle. Although the assumed space charge parameter is relatively small many resonances can be seen after 50000 revolutions, i.e. about 1 secand of storage time. The dependence on the crossing angle and on the order of the satellite resonances is investigated.

\section{Introduction}

Satellite resonances which are excited by the beam-beam interaction at a crossing angle were first investigated in the $\mathrm{e}^{+} e^{-}$storage ring OORIS I $[1,2]$. The resonance condition is given by:

$$
Q_{B}=\frac{p+r Q_{s}}{q}
$$

where $Q_{8}$ and $Q_{5}$ are the betatron (horizontal or vertical) and the synchrotron frequency, respectively, and $p, r$ and $a$ are integers. Beam losses were explained with the help of computer simulations and special measurements with single bunches in both beams which showed 12 satellite resonances in the neighbourhood of the working point. Although the width of the resonances was very small (in the order of $10^{-4}$ ) and the distance between them relatively large the resonances limited the luminosity. In this case the limitation was also caused by an rf-quadrupole and a decoupling transmitter which gave to different bunches different betatron and synchrotron frequencies in order to suppress multibunch instabilities. Therefore always some of the $2 x$ 120 bunches were lost due to a satellite resonance.

In the first proposal for the e-p storage ring HERA [3] a crossing angle was foreseen since it has some advantages over head-on collision, e.g. less synchrotron radiation hits the detectors and the optics for the two rings are completely independent. It was assumed that with a smaller space charge parameter $(\xi=$ 0.0006 instead of 0.01 in Doris I) and without spread in betatron and synchrotron frequencies a good working point for the protons could be found.

In order to estimate the strength of the satellite resonances for protons computer simulations were made. Computer simulations are more time consuming for protons than for electrons and the results are not quite so satisfactory. Whereas in the case of electrons a simulation for a few damping times, i.e. less than one second storage time is sufficient to show the dangerous resonances, in the case of protons a simulation for several seconds cannot, in general, predict the lifetime which should be in the order of many hours. Only in the case when the proton amplitudes increase by a few percent or more during one second can one draw conclusions and expect a short lifetime. The simulation has, in fact, shown many of those dangerous resonances which gave an increase of amplitudes up to a factor of 6 .

\section{The simulation program}

For the calculation of the change of the proton coordinates due to the beam-beam interacton the thin lens approximation is made, i.e. the kick formalism is

* On Teave from DESY, Hamburg. applied. The kick of a particle which passes through a Gaussian bunch at an angle of $2 \phi$ was calculated in $[4,5]$. The four integrals over space charge density and time can be reduced to one $[4,6]$ and assuming relativistic proton energies ( $y \gg 1)$ and a smal] horizontal crossing angle $\left(\phi^{2} \ll 1\right)$ one obtains for the horizontal and vertical kick:

$$
\begin{aligned}
& \left(\begin{array}{c}
\Delta x^{\prime} \\
\Delta z^{\prime}
\end{array}\right)=\frac{2 \pi}{1-v} \int_{0}^{1-v^{2}} \exp \left\{-a w-\frac{b w}{1-w}\right\}\left(\begin{array}{c}
x_{e f} E_{x} / \beta_{x}{ }^{\star} \\
\frac{z \xi_{z} / \beta_{z}^{*}}{1-w}
\end{array}\right) \frac{d w}{\sqrt{1-w}} \\
& v=\frac{\sigma_{z}}{\sigma_{x e f}} \\
& a=\frac{1}{2} \frac{x_{\text {ef }}^{2}}{\sigma_{x e f}^{2}-\sigma_{z}^{2}}, \quad b=\frac{1}{2} \frac{z^{2}}{\sigma_{x e f}^{2}-\sigma_{z}^{2}} \\
& \xi_{x, z}=\frac{r_{0} N_{b}{ }^{\star} x_{2} z}{2 \pi y \sigma_{x e f, z}\left(\sigma_{x e f}+\sigma_{z}\right)} \\
& \sigma_{x e f}=\sqrt{\sigma_{x}^{2}+\phi^{2} \sigma^{2} s}, \quad x_{e f}=x+\phi s
\end{aligned}
$$

$x, z, s=$ proton coordinates, $\sigma_{x, z} s=$ standard deviation of horizontal, vertical and longitudinal electron distribution, $\beta^{\star} x, z=$ horizontal and vertical amplitude function for protons at the interaction point, $N_{b}=$ number of electrons per bunch, $r_{0}=$ classical proton radius, $\gamma=$ relative proton energy. Here it is assumed that $\sigma_{x e f}>\sigma_{z}$. At the same time the proton energy is changed by [7]:

$$
\frac{\Delta E}{E}=\phi \Delta x^{\prime}
$$

The integral was solved numerically for $150 \times 150$ points with a spacing of 0.2 in both $\sigma_{x e f}$ and $\sigma_{z}$. For each passage of a proton, $\Delta x^{\prime}$ and $\Delta z^{\prime}$ are interpolated quadratically [8]. The longitudinal motion of the interaction point, seen by a proton due to its phase oscillation, varies the betatron phase advance between interaction points and changes the kicks $\left(\beta^{*} x^{\Delta x^{\prime}}\right.$ and $\beta^{*} z^{\Delta z}$ ) if the amplitude functions $\beta^{*} x, z$ at the interaction are not equal. The first part which is equivalent to the chromaticity and which increases linearly with the bunch length is always taken into account. The second part which increases quadratically with the bunch length is neglected. Between the interaction points the betatron and synchrotron coordinates are transformed linearly.

Most of the simulations were done for 50000 revolutions, i.e. about 1 second storage time. The precision of the calculation was about $10^{-14}$. Back-tracking gave the initial coordinates to within an accuracy of $10^{-3}$. Only for more than 200000 revolutions, back-tracking gave relative deviations from the initial values larger than 1 (only on resonances).

The following HERA-parameters were used:

$2 \phi=10 \mathrm{mrad}, \xi_{X}^{+}=0.0006, \xi_{Z}^{+}=0.0009, \sigma_{x}^{+}=0.12 \mathrm{~mm}$, $\sigma_{Z}^{+}=0.027 \mathrm{~mm}, \sigma_{\bar{x}}=0.22 \mathrm{~mm}, \sigma_{\bar{z}}=0.01 \mathrm{~mm}, \beta_{x}^{*}=3 \mathrm{~m}$, $\beta_{z}^{*}=0.3 \mathrm{~m}, \quad \sigma_{S}^{+}=95 \mathrm{~mm}, \quad \sigma_{\bar{S}}=9 \mathrm{~mm}, \quad Q_{S}^{+}=0.0161$.

The crossing angles are assumed to have alternating signs. 


\section{Simulation results}

The first figure shows the increase of amplitudes during 50000 revolutions on the resonance $4 n+$ $\left(10+50_{s}\right) / 3$ where $n$ is an arbitrary integer. The lower curve gives the maximum rms value of the horizontal coordinates of the 64 particles averaged over 1000 revolutions. The upper curve gives the maximum amplitude which appeared during the 50000 revolutions for a single particle. Both curves have a very small width (in the order of $10^{-4}$ ) which is in agreement with measurements in DORIS I. It is caused by the nonlinear tune shift of the beam-beam interaction. When the amplitudes increase due to the resonance excitation the frequency decreases due to the non-linear tune shift and the particles come out of resonance. Therefore, the amolitudes can increase considerably only in a region where the non-linear tune shift is weak, i.e. at large amplitudes.

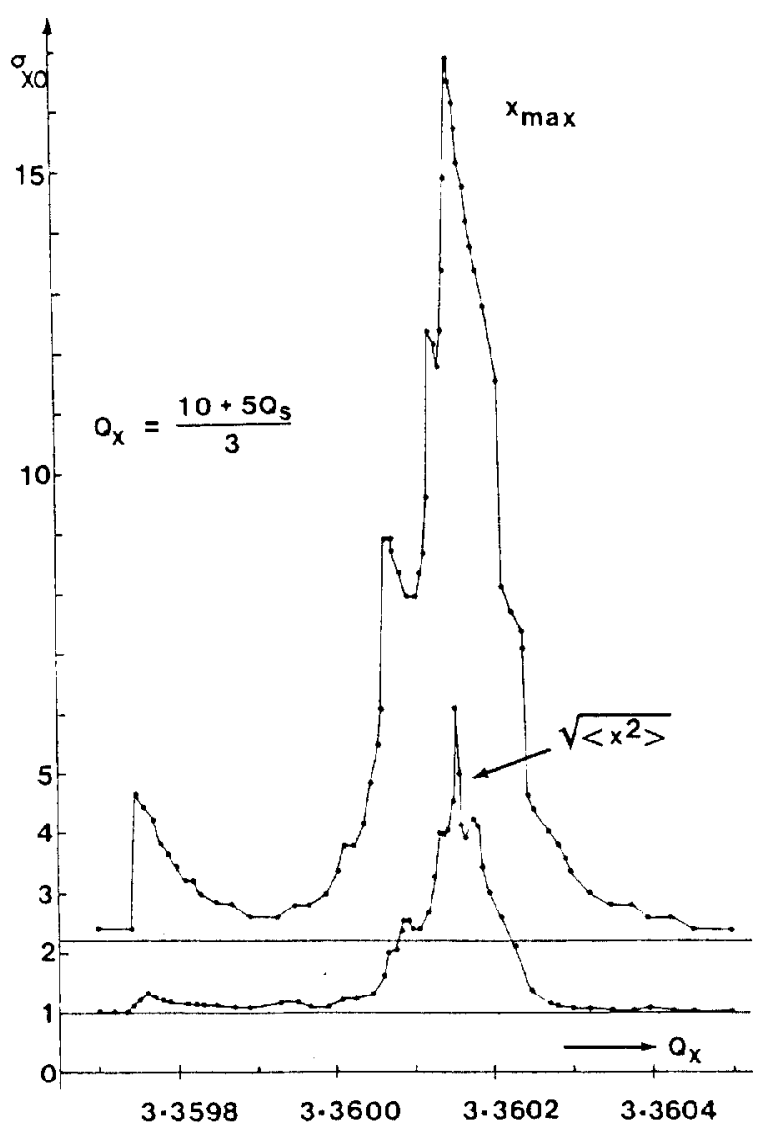

Fig.1 - Dependence of the maximum betatron amplitude and the beam width on the betatron frequency.

Fig. 2 shows the dependence of the maxima of the two curves on the crossing angle. A strong and almost linear increase of the amplitudes begins with crossing angles of the order of $\sigma_{\bar{x}} / \sigma_{\mathrm{S}}^{+}$, i.e. in this case at about $1.4 \mathrm{mrad}$. At this crossing angle protons at $s= \pm \sigma_{S}^{+}$, pass the center of the other bunch at a distance of $\sigma \bar{x}$ and come into the non-linear region of the space charge forces where satellites of higher order can be excited.

Fig. 3 shows all satellites of the resonance $Q_{B}=4 n+10 / 3$. In the case of satellite resonances the difference resonances are stronger than the sum reso-



Fig.2 - Dependence of the maximum amplitude and the maximum beam width on the crossing angle.

nances [1]. On a difference resonance the difference of the betatron and synchrotron oscillation energies remains constant. Therefore both amplitudes can increase as long as there are no other limitations. On a sum resonance, however, the sum of the oscillation energies remains constant, i.e. both amplitudes are limited. Since in the case of beam-beam interaction the increase of amplitudes is mainly limited by the non-linearity of the tune shift, the two kinds of resonances give almost the same increase for higher order satellites and for large space charge parameters.

Fig. 3 shows also that only odd satellites are excited. This happens when the centers of the two bunches collide since in this case the potential of the space charge farces is completely symmetric. Then, only those satellites can be excited [1], for which $q+r$ is even. If the two bunches are horizontally displaced the potential is no longer symmetric and both even and odd satellites can be excited. Also the symmetry of the distribution of the interaction points suppresses many resanances. With alternating signs of equal crossing angles only those resonances are excited for which $2 p / N_{j}$ is odd where $N_{j}$ is the number of interaction points. For equal signs this condition is given by $\mathrm{p} / \mathrm{N}_{\mathrm{i}}=$ integer. The symmetry of the interaction points is destroyed by different betatron phase advances between the interaction points. This can be caused, e.g. by orbit displacements in sextupoles.

Simulations have shown satellites of non-linear resonances up to the order 11. For these resonances the rms value does not show an increase, however, the maximum amplitude increases by more than $10 \%$. 


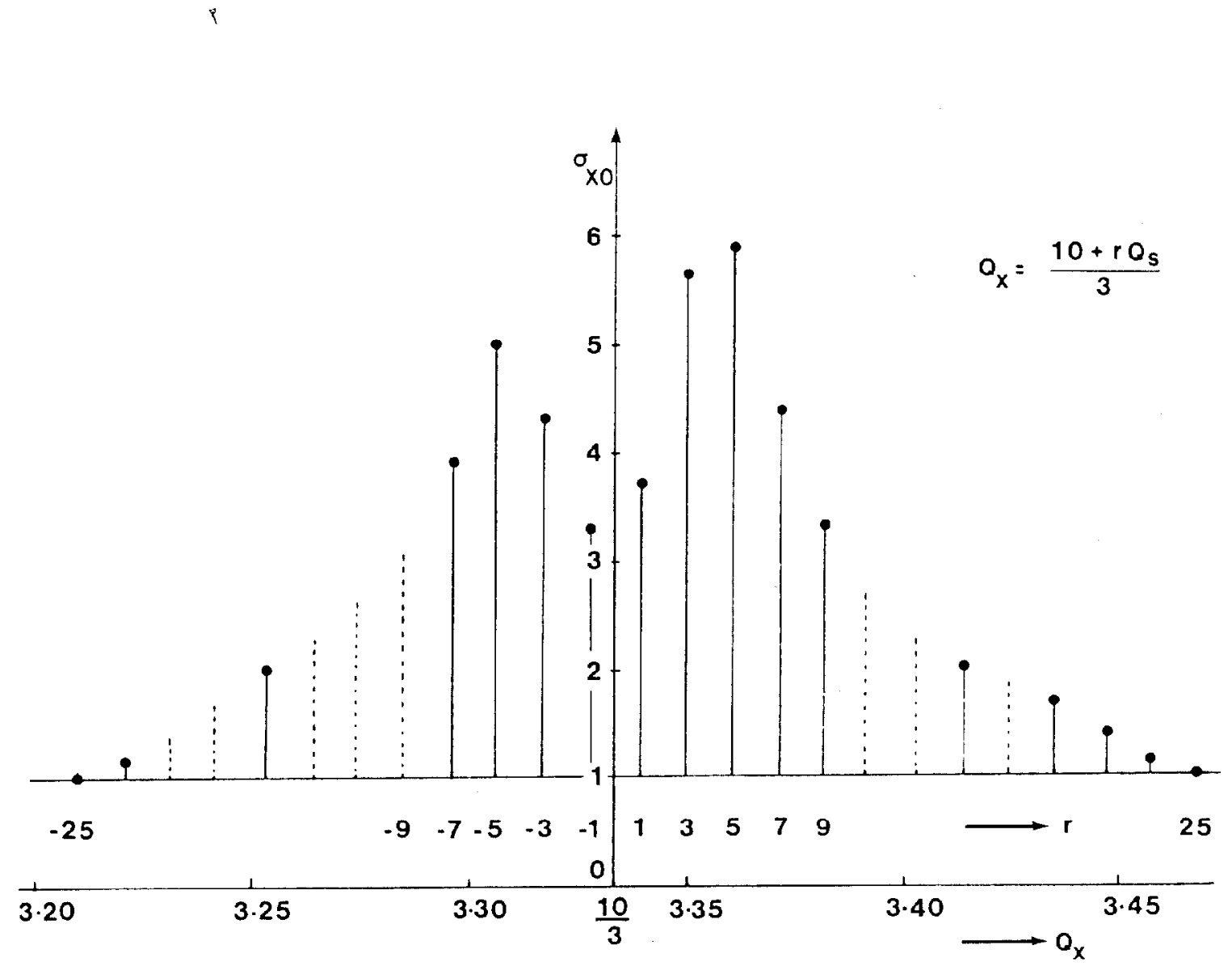

Fig.3 - Maximum beam width on all satellites of the resonance $Q_{X}=10 / 3$ (_- simulated, -...- estimated)

\section{Conclusions}

The simulation for protons in HERA with a crossing angle has shown many satellite resonances of very high order. The number of resonances is larger in HERA than in DORIS I since there is no damping for protons and since the ratio $\phi \sigma_{s} / \sigma_{x}$ which is a measure for the strength of this effect is 4 for HERA and only 0.5 for DORIS I. All these resonances appear in 1 second, and one must expect still more resonances after some hours. An experiment in the SPS [9] could not show the long time behaviour since the crossing angle $(2 \times 170$ urad) and the synchrotron frequency $\left(0_{S}=0.005\right)$ were too small. In the case of HERA with a crossing angle of $2 \times 10 \mathrm{mrad}$ and a synchrotron frequency of $\mathrm{Q}_{\mathrm{s}}=0.016$ the large number of satellite resonances and the small distance between them will make it very difficult to find a working point with a sufficiently large lifetime.

\section{Acknowledgements}

The author is very grateful to J. Kewisch and $H$. Wagner from DESY and R. Keyser from CERN for their help in setting up and optimizing the simulation program.

\section{REFERENCES}

[1] A. Piwinski, Limitation of the luminosity by satellite resonances, DESY $77 / 18$ (1977).

[2] A. Piwinski, Satellite resonances due to beam-beam interaction, 1977 Particle Acc. Conf., Chicago, IEEE Trans. On Nuclear Science, Vol. NS-24, No. 3 June 1977.

[3] HERA, A proposal for a large electron-proton colliding beam facility at DESY, DESY HERA $81 / 10$ (1981)

[4] A. Piwinski, Der Raumlandungseffekt bei vertikalem oder horizontalem Kreuzungswinkel, Internal Report DESY-HI/1 (1969).

[5] A. Piwinski, Space charge effect with crossing angle, Nuclear Instr. and Meth. 81 (1970)

[6] A. Talman, Measurement, analysis and modification of multi-particle phenomena in accelerators, CLNS-84/610 (1984).

7] J.E. August in, Note Interne 35-69 (1969).

[8] A. Piwinski, Computer simulation of the beam-beam interaction, DESY 80/131 (1980).

[9] L.R. Evans, R.A. Faugier, R. Schmidt, Beam separation at the CERN SPS collider, this conference. 